\title{
ReaR

\section{Bloqueo epidural alto por migración de un catéter paravertebral colocado mediante visión directa del cirujano para control analgésico tras toracotomía}

Insausti Pacheco I, Martínez García E, Roca Amatria G, Moret Ruiz E.

Hospital Germans Trias I Pujol, Badalona, Barcelona.

\section{Resumen}

La técnica analgésica de elección para el control del dolor postoperatorio tras una cirugía de tórax sigue siendo la analgesia epidural. En los últimos 10 años, varios estudios han presentado al bloqueo paravertebral continuo como igualmente efectivo e incluso más seguro con pocas complicaciones graves asociadas. Presentamos el caso de una paciente de 73 años, ASA III, sometida a cirugía de resección pulmonar con colocación de un catéter paravertebral por visión directa del cirujano para analgesia postoperatoria.

5 horas más tarde la paciente presentó clínica de bloqueo epidural alto (sensitivo hasta T1, motor hasta T4). Se detuvo la infusión de anestésico local, y se confirmó la localización de la punta del catéter mediante imagen radiológica con contraste. En este caso, la migración del catéter cuestiona la sensación de mayor seguridad de esta técnica y hace énfasis en la necesidad de mantener una monitorización estricta de cualquier procedimiento que realicemos.

\section{Introducción}

La técnica analgésica de elección para el control del dolor postoperatorio tras una cirugía de tórax sigue siendo la analgesia epidural. En los últimos 10 años, varios estudios han presentado al bloqueo paravertebral continuo como igualmente efectivo e incluso más seguro con pocas complicaciones graves asociadas. Presentamos el caso de una paciente de 73 años, ASA III, sometida a cirugía de resección pulmonar con colocación de un catéter paravertebral por visión directa del cirujano para analgesia postoperatoria.

5 horas más tarde la paciente presentó clínica de bloqueo epidural alto (sensitivo hasta T1, motor hasta T4). Se detuvo la infusión de anestésico local, y se confirmó la localización de la punta del catéter mediante imagen radiológica con contraste. En este caso, la migración del catéter cuestiona la sensación de mayor seguridad de esta técnica y hace énfasis en la necesidad de mantener una monitorización estricta de cualquier procedimiento que realicemos.

La toracotomía en la cirugía de resección pulmonar se asocia a un intenso dolor postoperatorio. Un control inadecuado del mismo puede provocar complicaciones postoperatorias, comprometiendo el resultado quirúrgico y aumentando la morbimortalidad.

El gold standard analgésico en este tipo de cirugías sigue siendo la analgesia epidural. Debido a los conocidos efectos secundarios asociados a esta técnica (retención urinaria, hipotensión, bloqueo motor, lesión neurológica potencial, fallo de la técnica etc.) y sus contraindicaciones en ciertos escenarios 
(tratamientos antiagregantes / anticoagulantes, dificultades anatómicas etc.), se han investigado posibles alternativas analgésicas.

El bloqueo paravertebral se describió por primera vez por Hugo Sellheim en 1905 pero fue en 1979 cuando Eason y Wyatt describieron la colocación de un catéter paravertebral (CPV) (1). Más recientemente, se ha descrito la colocación mediante ecografía o visión directa del cirujano, lo que incrementaría la tasa de éxito. En los últimos 20 años se han publicado muchos estudios. Uno realizado por Federico Raveglia y otros (2) en 2014 comparó ambas técnicas (grupos epidural y paravertebral) para la analgesia tras toracotomía. Las conclusiones fueron que el efecto analgésico era similar, pero sin observarse efectos adversos en el grupo de bloqueo paravertebral.

Se han publicado algunos casos de eventos graves asociados a la colocación percutánea de un CPV, como el desplazamiento subdural (3) o epidural (4) (5) (6) del mismo. Sin embargo, no hay publicaciones sobre el desplazamiento y difusión de anestésico local (AL) a nivel epidural tras la colocación por visión directa por parte del cirujano.

Presentamos este caso para describir un evento no descrito previamente en una paciente sometida a cirugía de resección pulmonar.

\section{Caso clínico}

Mujer de 73 años, ASA III, programada para una resección pulmonar atípica en los lóbulos superior e inferior izquierdos (LSI y LII) por metástasis de un cáncer de recto tratado previamente con quimioterapia neoadyuvante, cirugía oncológica y radioterapia adyuvante.
Bajo monitorización estándar (pulsioximetría, presión arterial no invasiva y electrocardiograma de 5 derivaciones) se intentó colocar un catéter epidural torácico siendo imposible por circunstancias anatómicas. Se completó la monitorización mediante un monitor de la profundidad de hipnosis (BIS®) y del bloqueo neuromuscular tipo TOF. Se realizó una anestesia general mediante $150 \mathrm{mg}$ de propofol, $100 \mathrm{mg}$ de succinilcolina (por sospecha de dificultad en el manejo de la vía aérea) y $150 \mathrm{mcg}$ de fentanilo endovenosos. Se llevó a cabo una intubación oral mediante un tubo endotraqueal del número 8 y se colocó un bloqueador bronquial izquierdo (Fuji uniblocker®) para la ventilación unipulmonar, sin incidencias. El mantenimiento se realizó mediante desfluorano (concentración alveolar mínima de 0,8), infusión de remifentanilo $\quad 0,1-0,2 \mathrm{mcg} / \mathrm{kg} / \mathrm{min}$ ) $\quad \mathrm{y}$ rocuronio. Se monitorizó finalmente la presión arterial invasiva en arteria radial. En decúbito lateral derecho se llevaron a cabo las resecciones atípicas de LSI y LII y linfadenectomía de la región 5 mediante toracotomía posterolateral. Antes de finalizar la cirugía, se colocaron un tubo de drenaje pleural de $28 \mathrm{Fr}$ y un catéter paravertebral (catéter epidural de $80 \mathrm{~mm}$ Portex Smith Medical) entre el $4^{\circ}$ y $5^{\circ}$ espacios intercostales mediante visión directa por parte del cirujano. Previos al despertar se administraron $7 \mathrm{mg}$ de morfina, dexketoprofeno 50mg, metamizol $2 \mathrm{~g} \quad \mathrm{y}$ ondasentrón $4 \mathrm{mg}$ endovenosos y se inició la perfusión mediante bomba elastomérica de bupivacaína $0,25 \%$ a $12 \mathrm{ml} / \mathrm{h}$ a través del catéter paravertebral. No hubo incidencias intraoperatorias y la paciente se extubó en quirófano sin problemas. Tras dos horas de vigilancia en la unidad de recuperación postanestésica, la paciente se fue de alta a planta. 
En las 2 primeras horas, la paciente estaba somnolienta, sin dolor y $\sin$ déficit neurológico aparente. $12 \mathrm{~h}$ después, la paciente manifestó parestesias y bloqueo motor en extremidades inferiores, confirmado por enfermería. La paciente fue entonces valorada por facultativos de la unidad del dolor demostrándose un bloqueo sensitivo hasta $\mathrm{T} 1$ y un bloqueo motor hasta $\mathrm{T} 4$, sin compromiso respiratorio ni hemodinámico. La perfusión de AL se detuvo de inmediato y se realizó un control radiológico mediante la inyección de contraste a través del CPV.

Se demostró la migración de la punta del catéter y la difusión de contraste al espacio epidural (Fig.1). Tras la comprobación de un recuento plaquetario y una coagulación normales, se retiró el catéter, y la paciente se mantuvo bajo observación hasta la reversión completa del bloqueo. Finalmente, la paciente fue dada de alta a planta sin mayores consecuencias.

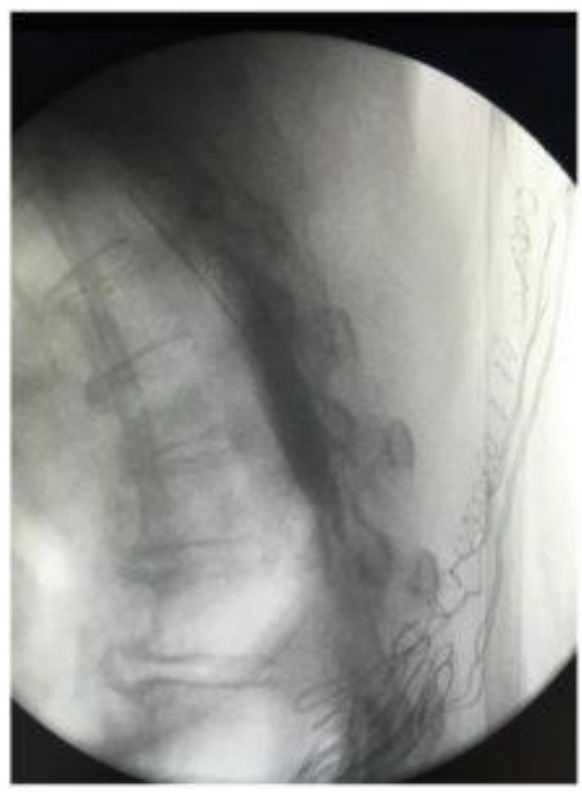

Fig.1 Imagen radiológica mostrando una visión lateral de la columna vertebral. Puede observarse la difusión paravertebral y epidural del contraste.

\section{Discusión}

Nuestra práctica diaria pretende ofrecer el mayor beneficio para los pacientes con la mayor seguridad en los procedimientos. Hasta ahora, los estudios que comparaban el bloqueo paravertebral continuo con la analgesia epidural para el control del dolor tras toracotomía se inclinaban a favor del primero (2) (7), ya que asocia un bloqueo simpático y sensitivo unilaterales con menos efectos secundarios, ofreciendo una analgesia equivalente. Esto es relevante ya que está aumentando el número de toracotomías en población cada vez mayor, más frágil y que asocia más comorbilidad. A pesar de todo, como toda técnica, el CPV no está libre de complicaciones consecuencias

(9) con potencialmente peligrosas descritas tras su introducción de forma percutánea.

Sin embargo, la ausencia de eventos graves descritos tras su colocación bajo visión directa por un cirujano genera una falsa sensación de seguridad que lleva a una vigilancia posiblemente menos estrecha que la aplicada para una infusión epidural. Este caso pretende mostrar una complicación no descrita tras la colocación por visión directa de un $\mathrm{CPV}$, que podría considerarse con el tiempo como la técnica de elección.

\section{Conclusión}

Cualquier procedimiento instrumental puede presentar complicaciones. Es crucial y necesario aplicar un control estricto basado en protocolos de actuación en la monitorización de cualquier técnica invasiva. Esto debería realizarse comprobando no solo la eficacia sino también la aparición de efectos adversos. 


\section{Bibliografía}

1.- Eason MJ, Wyatt R. Paravertebral thoracic block- A reappraisal. Anaesthesia. 1979; 34:

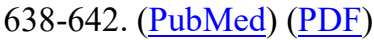

2.- Raveglia F, Rizzi A, Leporati A, Di Mauro P, Cioffi U, Baisi, A. Analgesia in patients undergoing thoracotomy: Epidural versus paravertebral technique. A randomized, doubleblind, prospective study. J Thorac Cardiovasc Surg. 2014;147: 469-74. (ubMed)

3.- Garutti I, Hervias M, Barrio JM, Fortea F, De la Torre F. Subdural Spread of Local Anesthetic Agent following Thoracic Paravertebral Block and Cannulation. Anesthesiology. 2003; 98:1005-7. ( (PDF)

4.- Frohm RM, Raw RM, Haider N, Boezaart AP. Epidural Spread After Continuous Cervical Paravertebral Block: A Case Report. Reg Anesth Pain Med. 2006; 31(3): 279-281. (PubMed)

5.- Yoshida T, Shimizu H, Furutani K, Baba H. Unintentional epidural placement of a thoracic paravertebral catheter inserted using an ultrasound-guided technique: a case report. J Anesth. 2016; 30 (4): 727-730. ( ( $\underline{\text { HTML) }}$

6.- Lucas SD, Higdon T, Boezaart AP. Unintended Epidural Placement of a Thoracic Paravertebral Catheter in a Patient with Severe
Chest Trauma. Pain Med. 2011; 12: 1284-1289. (HTML) (PDF)

7.- Kotzé A, Scally A, Howell S. Efficacy and safety of different techniques of paravertebral block for analgesia after thoracotomy: a systematic review and metaregression. $\mathrm{Br} \mathrm{J}$ Anaesth 2009; 103: 626-36. (ubMed) (NML)

8.- Karmakar MK, Kwok WH, Kew J. Thoracic paravertebral block: radiological evidence of contralateral spread anterior to the vertebral bodies. $\mathrm{Br} \mathrm{J}$ Anaesth. 2000; 84: 263-5. (PubMed) $(\underline{\text { PDF }})$

9.- Luyet C, Hermann G, Ross S, Vogt A, Greif R, Moriggl B. et al. Ultrasound-guided thoracic paravertebral puncture and placement of catheters in human cadavers: where do catheters go? Br J Anaesth. 2011; 106 (2): 246-54. (PubMed) ( $\underline{\text { HTML) }}$

Correspondencia al autor

Itziar Insausti Pacheco

iinsaustip@gmail.com

Residente de anestesiología y reanimación. Hospital Germans Trias I Pujol, Badalona, Barcelona.

Aceptado para el blog en marzo de 2019. 